\title{
LINEAR STABILITY ANALYSIS AND DIRECT NUMERICAL SIMUALATION OF DOUBLE-LAYER RAYLEIGH-BÉNARD CONVECTION
}

\author{
É. FONTANA ${ }^{1}$, E. MANCUSI ${ }^{1,2}$, A. A. ULSON DE SOUZA ${ }^{1}$, S. M. A. GUELLI U. SOUZA ${ }^{1}$ \\ ${ }^{1}$ Universidade Federal de Santa Catarina, Departamento de Engenharia Química e Engenharia de \\ Alimentos \\ ${ }^{2}$ Università del Sannio, Facoltà di Ingegneria \\ E-mail para contato: eliton.fontana@gmail.com
}

\begin{abstract}
Natural convection in superimposed layers of fluids heated from below is commonly observed in many industrial and natural situations, such as crystal growth, coextrusion processes and atmospheric flow. The stability analysis of this system reveals a complex dynamic behavior, including potential multiplicity of stationary states and occurrence of periodic regimes. In the present study, a linear stability analysis (LSA) is performed to determine the convection onset as a function of imposed boundary conditions, geometrical configuration and specific perturbations with different wavenumbers. To investigate the effects of the non-linear terms neglected by the linear stability analysis, a direct numerical simulation of the full nonlinear problem is performed with the use of CFD techniques. The numerical simulations results show an excellent agreement with the LSA results near the convection onset and an increase in the deviation as the Rayleigh number increases beyond the critical value.
\end{abstract}

\section{INTRODUCTION}

Single layer Rayleigh-Bénard (RB) convection is one of the most widely studied systems in the transport phenomena field, mainly because the large number of applications and the relative simplicity of the governing equations. This system represents a natural convection condition occurring in a horizontal layer of fluid where energy is added from below and removed from above, giving rise to cellular structures called Bénard cells (or convective cells) when the buoyancy forces are sufficiently stronger than the viscous forces.

The theoretical basis of this phenomenon was presented by Lord Rayleigh in 1916, based on experimental results realized by Bénard 16 years before. Rayleigh showed that the system stability is governed by a dimensionless parameter, later known as the Rayleigh number (Ra), which represents precisely the ratio between buoyancy and viscous forces. Whenever the Rayleigh number is below a certain critical value, the heat transfer will occur basically by conduction. However, when this critical value is exceeded the system becomes unstable and evolves to a different equilibrium state where 


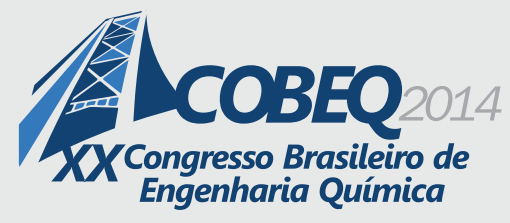

19 a 22 de outubro de 2014
Florianópolis/SC

natural convection is the main heat transfer mechanism. In a bifurcation theory terminology, in this condition a bifurcation occurs and the critical point correspond to a bifurcation point. Through linear stability analysis, it is possible to define the critical Rayleigh values $\left(R a_{c}\right)$, as well as the wavenumber associated with the perturbation that first turns the system unstable $\left(\alpha_{c}\right)$. As presented by Pellew and Southwell (1940), for unbound two-dimensional systems with Newtonian fluids, the critical parameters depend only upon the boundary conditions applied to the velocity vectors at both horizontal boundaries, which can be treated as rigid walls or free surfaces. The values obtained considering the possible combinations are summarized in Table 1, showing that the presence of a rigid wall significantly increases the critical parameters.

Table 1 - Critical parameters for single layer Rayleigh-Bénard convection (Pellew and Southwell, 1940)

\begin{tabular}{c|c|c}
\hline Boundary condition & $R a_{c}$ & $\alpha_{c}$ \\
\hline Rigid - rigid & 1707.8 & 3.117 \\
Rigid - free & 1100.7 & 2.682 \\
Free - free & 657.5 & 2.221 \\
\hline
\end{tabular}

The presence of a second fluid layer significantly increases the system complexity and can affect the stability in many ways, as for example through competition of convective modes in each layer, control of one layer over the other one, interface deformation and unstable convective modes controlled by interfacial tension gradients. Due to this complex dynamical behavior and the large number of governing parameters, the stability of double layer Rayleigh-Bénard convection is still poorly understood. Despite this, as mentioned by Anderek et al. (1998), systems where stratification occurs due to differences in density or thermal properties are fairly common in the analysis of geophysical systems, atmospheric flow, astrophysics and industrial processes. The study of this system was originally motivated by the hypothesis that the Earth's mantle is stratified as a result of seismic discontinuity observed at a depth of approximately $660 \mathrm{~km}$. Examples of technological applications where double-layer RB convection can take place are liquid encapsulated technics for crystal growth (Li et al., 2009), glass and dispersion materials processing (Prakash, 1997) and microchannel chemical reactors (Fudym et al., 2007).

The stability analysis of double-layer RB convection needs to consider several mechanisms that can turn the system unstable, and in most of the cases these mechanisms are associated with substantially different time and length scales. Therefore, a global analysis considering all the possible mechanisms can not be easily reached using a single method. Among the most used methods of hydrodynamic stability analysis, the linear analysis using normal modes has been receiving much attention in the past decades due to their relative simplicity and flexibility. However, this method has intrinsic limitations derived by the fact that high order terms are neglected due to the linearization process. In the present study, direct numerical simulation (DNS) of the full non-linear set of governing equations using modern computational fluid dynamic (CFD) based techniques will be performed and compared to the results obtained through linear stability analysis (LSA). 


\section{MATHEMATICAL MODEL}

A scheme of the geometrical configuration considered is shown in Figure 1. The system consists of two layers of immiscible fluids confined between horizontal rigid walls. The system is assumed to be unbound in the $z$ and $x$-directions; however perturbations in these directions are also included in the model. The thickness of the bottom layer, $d_{1}$, is used as length scale, so that the bottom wall is placed at the dimensionless position $y=-1$ and the upper wall is placed at $y=$ $d_{2} / d_{1}=d_{0}$. Both walls are considered to be at constant temperatures and we will limit our analysis to the case that $T_{H}>T_{C}$ will be analyzed. The non-deformed interface is positioned at $y=0$ and the interface deformation is evaluated by the function $\eta=\eta(x, z, t)$.

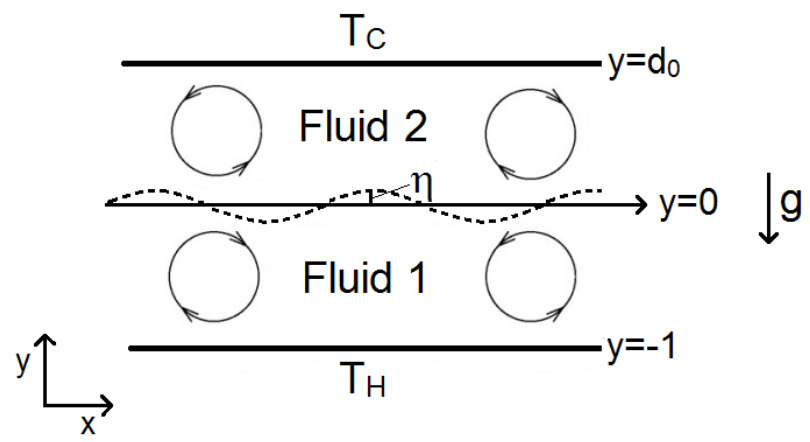

Figure 1 - Physical domain

Each layer has its own set of governing equations, being these equations linked by the boundary conditions at the interface. It is assumed that the flow in both layers is incompressible, the fluids have Newtonian behavior and the Boussinesq approximation is valid for the entire range of conditions evaluated. In this condition, the governing equations are the standard Navier-Stokes and energy and mass conservation equations. Boundary conditions of no-slip and no-penetration are applied to the velocity vector at the solid walls $\left(y=-1\right.$ and $\left.y=d_{0}\right)$, while fixed temperatures are defined at the same locations. At the interface $(y=\eta)$, conditions of normal and tangential velocity continuity, as well shear and normal stress balance are used to determine the velocity field, while for the energy conservation equation conditions of temperature and heat flux continuity are assumed.

In order to characterize the stability proprieties of a given base state through linear stability analysis, the variables in the set of governing equations are expressed as a sum of the base state and an infinitesimal perturbation, so that the dynamic behavior of these perturbations can be used to define the state stability: if the perturbations decrease over time and eventually disappear the system will be stable, otherwise it will be unstable. In the linear stability analysis only infinitesimal perturbations are considered, so that all the high order terms can be neglected. Moreover, nondimensional variables will be introduced by using $d_{1}$ as length scale, $\kappa_{1} / d_{1}$ as a scale to the perturbation velocity, $\rho_{1} v_{1} \kappa_{1} / d_{1}^{2}$ to the pressure and $\psi_{1} d_{1}$ as a scale to the temperature, where $\kappa_{1}, \rho_{1}$ and $v_{1}$ are, respectively, the thermal diffusivity, density and kinematic viscosity of the bottom layer and $\psi_{1}$ is the static temperature gradient between the non-deformed interface and the bottom wall. 
The base state represents a solution to the set of governing equations and the related boundary conditions. To determine the onset of natural convection in a double layer RB convection system, the base state corresponds to the stationary fluid with a linear temperature profile associated with heat transfer only by conduction. The equilibrium point can be considered stable if is stable respect to any possible infinitesimal perturbation, differently it is unstable. As described by Chandrasekhar (1961), one way to analyze the stability is express the perturbed variables in terms of normal modes. For example, a generic variable $k$ can be expressed as:

$$
k(x, y, z, t)=\bar{k}(y) e^{i(\alpha x+\beta z-\omega t)}
$$

Where $\bar{k}(y)$ is the eigenfunction associated to the original variable, $\alpha$ and $\beta$ are real wavenumbers in the $x$ and $z$-directions, respectively, and $\omega=\omega_{r}+i \omega_{i}$ is the wave velocity, where $\omega_{r}$ is the phase velocity and $\omega_{i}$ the perturbation temporal growth rate. A condition when $\omega_{i}<0$ represents a dumped perturbation, while when $\omega_{i}>0$ the perturbation will grow and so the base state will be unstable. The condition $\omega_{i}=0$ is called neutral stability.

After some manipulation the pressure can be eliminated and the equations governing of the system stability in the bottom layer can be expressed as:

$$
\begin{aligned}
& -\frac{i \omega}{\operatorname{Pr}}\left(\frac{d^{2} \phi_{1}}{d y^{2}}-k^{2} \phi_{1}\right)=\frac{d^{4} \phi_{1}}{d y^{4}}-2 k^{2} \frac{d^{2} \phi_{1}}{d y^{2}}+k^{4} \phi_{1}-k^{2} R a \theta_{1} \\
& -i \omega \theta_{1}-\phi_{1}=\frac{d^{2} \theta_{1}}{d y^{2}}-k^{2} \theta_{1}
\end{aligned}
$$

where $\phi_{1}$ and $\theta_{1}$ represent the perturbation of the vertical velocity and temperature, respectively, $k^{2}$ is defined as $k^{2}=\alpha^{2}+\beta^{2}$ and the Prandtl and Rayleigh numbers are given by:

$$
\operatorname{Pr}=\frac{v_{1}}{\kappa_{1}} \quad R a=\frac{\beta_{1} g \psi_{1} d_{1}^{4}}{v_{1} \kappa_{1}}
$$

where $\beta_{1}$ is the coefficient of thermal expansivity of the bottom layer and $g$ is the gravitational acceleration. The equations for the upper layer (denoted by the subscript 2) can be expressed as:

$$
\begin{aligned}
& -\frac{i \omega}{\operatorname{Pr}}\left(\frac{d^{2} \phi_{2}}{d y^{2}}-k^{2} \phi_{2}\right)=\frac{v_{2}}{v_{1}}\left(\frac{d^{4} \phi_{2}}{d y^{4}}-2 k^{2} \frac{d^{2} \phi_{2}}{d y^{2}}+k^{4} \phi_{2}\right)-\frac{\beta_{2}}{\beta_{1}} k^{2} R a \theta_{2} \\
& -i \omega \theta_{2}-\frac{\psi_{2}}{\psi_{1}} \phi_{2}=\frac{\kappa_{2}}{\kappa_{1}}\left(\frac{d^{2} \theta_{2}}{d y^{2}}-k^{2} \theta_{2}\right)
\end{aligned}
$$

For the sake of brevity, the boundary conditions will not be presented here, being the reader directed to the studies of Rasenat et al. (1988) and Cardin et al. (1992) for more details. It is sufficient to say that three dimensionless numbers appear in the final expressions: the Marangoni $(M a)$ and 
Schmidt $(S)$ numbers and a Rayleigh number based on the density difference between the upper and bottom layers $\left(R a_{\rho}\right)$. These numbers are defined as:

$$
M a=-\frac{d \gamma}{d T} \frac{\psi_{1} d_{1}^{2}}{v_{1} \rho_{1} \kappa_{1}} \quad S=\frac{\gamma d_{1}}{d_{1} \rho_{1} \kappa_{1}} \quad \operatorname{Rap}=\frac{g d_{1}^{3}}{v_{1} \kappa_{1}}\left(1-\frac{\rho_{2}}{\rho_{1}}\right)
$$

where $\gamma$ is the interfacial tension and $T$ is the temperature.

To solve the generalized eigenvalue problem, a pseudo-spectral method using Chebyshev polynomials was applied. The DNS analysis based on CFD techniques was performed using the FLUENT 14.0 software, where the set of governing equations is discretized through a finite volume approach. Appropriated mesh size and control parameters were used. To evaluate the interface deformation the Volume of Fluid (VOF) method was used. More details about this method can be found in Fontana et al. (2013) and Mancusi et al. (2014).

\section{RESULTS}

The physical properties were chosen so that the Rayleigh number in both layers is the same when $d_{0}=1$. Moreover, the conditions $M a=S=0$ (no thermo-capillary effect) and $\operatorname{Pr}=1$ are assumed and unless otherwise mentioned, $R a_{\rho}=10^{5}$. In this condition the interface deformation can be neglected.

Figure 2 shows the stability limit curve obtained through LSA, where the critical Rayleigh number is presented as a function of the depth of the upper layer $\left(d_{0}\right)$. Moreover, the points obtained by CFD simulation for several $d_{0}$ values are reported in Fig. 2 too. These points classified as stable or unstable depending upen by the presence or not of convective motion.

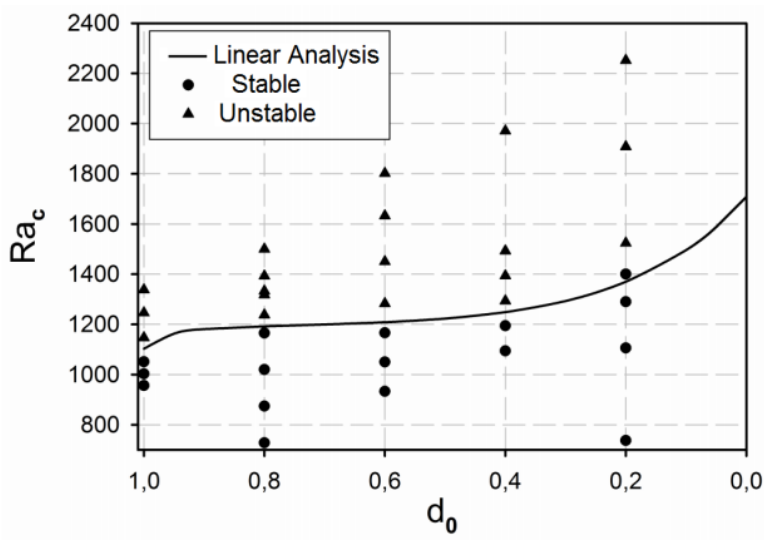

Figure 2 - Comparison between stability limits obtained through LSA (line) and DNS (points).

As can be seen in Figure 2, the limits defined by the linear stability analysis are in excellent 
agreement with the results of the CFD simulations, with only one stable point appearing in the unstable region. However, in the limit of the critical value the convection onset is not easily defined in the CFD simulations, since an infinite theoretically time would be necessary to develop a stationary convective state. It is worth to mention that the $R a_{c}$ values obtained in the cases $d_{0}=0$ and $d_{0}=1$ correspond exactly to the values of critical Rayleigh number for single layer RB convection when, respectively, conditions of rigid - rigid walls and rigid wall - free surface are considered (see Table 1). This shows a complete consistence between the double and single-layer models.

Besides the convection onset, the LSA allows to obtain the spatial distribution of the velocity and temperature fields through eigenfunctions. A comparison between these eigenfunctions and the actual velocity and temperature profiles (deviation from the base state) obtained by the CFD simulations are shown in Figure 3 for $d_{0}=0.8$ and $R a$ close to the critical value.

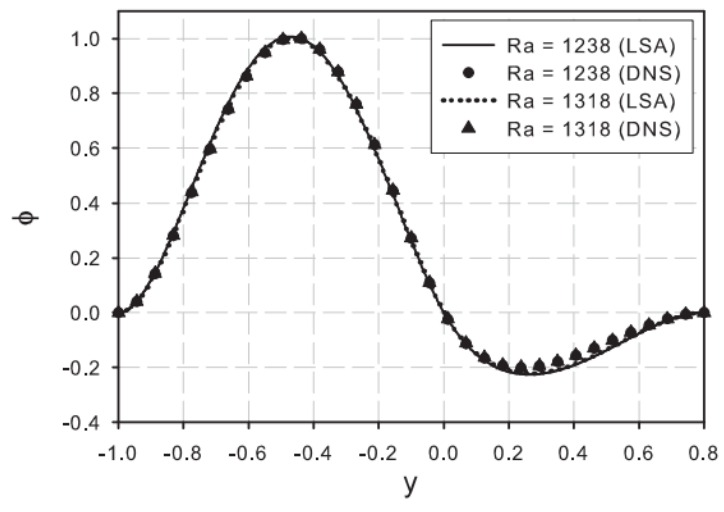

(a) Vertical velocity

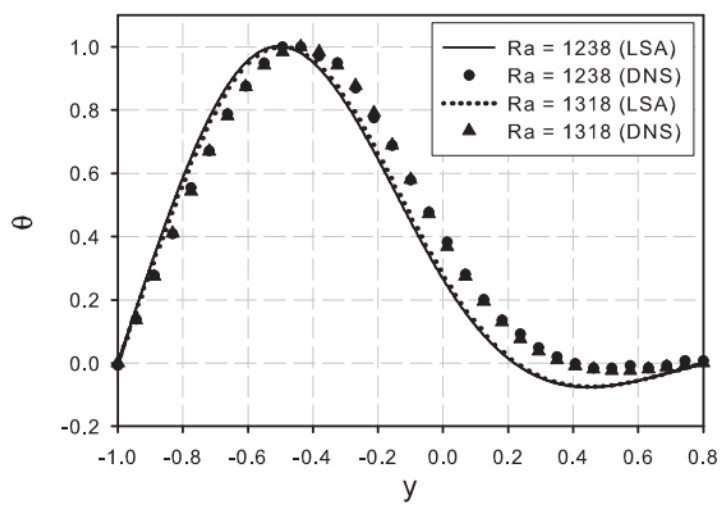

(b) Temperature

Figure 3 - Comparison between eigenfunctions obtained through LSA and velocity and temperature profiles obtained with CFD simulations.

The profiles obtained by LSA and the CFD simulation of full nonlinear problem show an excellent agreement, in particular the velocity profiles. Despites a small deviation in the magnitude in comparison with the CFD results, the LSA eigenfunctions of temperature deviation shows the same tendency. The small change in Rayleigh number does not affect significantly the profiles in any case.

One of the most restrictive hypothesis of the LSA using normal modes is that the interface deformation is not strong enough to a point where the high order terms cannot be neglected. In other words, this condition can be expressed as $\eta \ll d_{1}$. The interface deformation is controlled by the normal stress balance, in particular by the value of $R a_{\rho}$. If this value is not large enough to keep the interface stable, surface waves will emerge and eventually the system undergoes to an unstable state. The condition $R a_{\rho}<0$ will not be considered since it represents, by definition, a Rayleigh-Taylor instability.

Vertical velocity and temperature deviation profiles, obtained through CFD simulations, are 
presented in Figure 4 for $R a_{\rho}=10^{3}$ and $d_{0}=1$, together with the interface position for several values of $R a>R a_{c}$. To facilitate the results visualization, allowing the use of a single legend, the values for $R a<1425$ were multiplied by a scale, as indicated in the figure for each case.
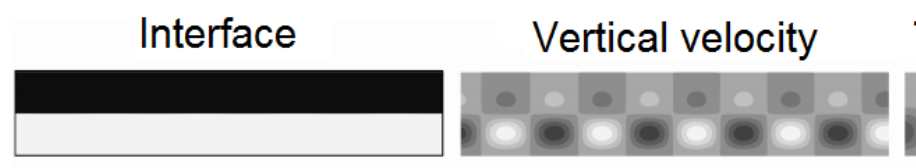

Temperature deviation

(a) $R a=1165(\times 25)$
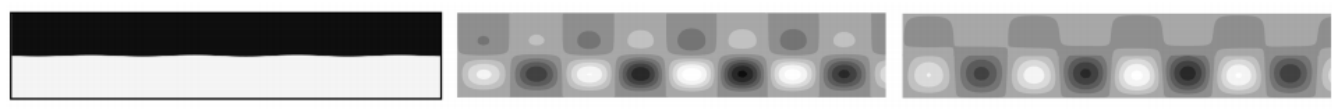

(b) $R a=1234(\times 7)$
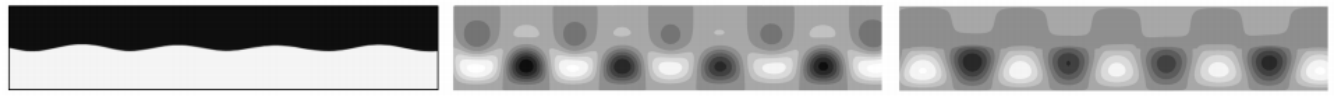

(c) $R a=1371(\times 1.5)$
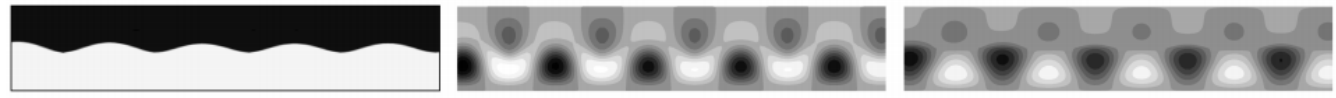

(d) $R a=1425$

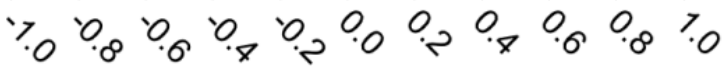

Figure 4 - Influence of interface deformation on velocity and temperature fields for $R a_{\rho}=10^{3}$.

For $R a_{\rho}=10^{3}$ the critical Rayleigh number is approximately 1163 , therefore the results shown in Figure 4-a are very close to the critical value. In this case, the interface deformation can be neglected and the velocity and temperature profiles obtained are similar to those obtained at higher $R a_{\rho}$ values. For $R a=1234$ (Figure 4-b), a small interface deformation can be observed, however the condition $\eta \ll d_{1}$ still holds and the velocity and temperature profiles are very similar to $R a=1163$.

In spite of the interface deformation be negligible for $R a$ near the critical value, as the Rayleigh number increases it becomes more evident, as can be seen in the Figure 4. For $R a=1371$ (Figure 4-c) the velocity is significantly altered and the convective cells are distorted. The interface has a wavy shape, with the points of maximum (crest) appearing in the region where the fluid ascends and the vertical velocity in the bottom layer is positive near the interface and the points of minimum appearing the descending region. For $R a=1425$ the deformations further increase and the deviation from the linearized model are more significant. 


\section{9 a 22 de outubro de 2014 \\ Florianópolis/SC}

\section{CONCLUSION}

In the present study a comparison between linear stability analysis using normal modes and direct numerical simulation with CFD techniques of double-layer Rayleigh-Bénard convection was presented. LSA techniques allow determining the critical value where the convection starts, however the linearization process neglects high order terms and this can lead to deviations from the actual system behavior. The CFD simulations, on the other hand, solve the full non-linear system of governing equation, but are much more computationally expensive. The results show that the LSA has an excellent performance near the critical point and so can be used to define the critical values. However, as the intensity of the convective motion increases the deviations from the linearized model increases, and the velocity and temperature profiles given by the LSA are not consistent anymore.

\section{REFERENCES}

ANDEREK, C. D.; COLOVAS, P. W.; DEGEN, M. M.; RENARDY, Y. Y. Instabilities in two layer Rayleigh-Bénard convection: Overview and outlook. Int. J. of Engineering Science, v. 36, p. 1451 1470, 1998.

CARDIN, P.; NATAF, H. C.; DEWOST, P. Thermal coupling in layered convection: evidence for an interface viscosity control for mechanical experiments and marginal stability analysis. J. of Physics II, v. 1, p. $599-622,1991$.

CHANDRASEKHAR, S. Hydrodynamic and Hydromagnetic Stability. [S.1.]: Clarendon Press, 1961.

FONTANA, É; MANCUSI, E.; SOUZA, A. A. U.; SOUZA, S. M. A. G. U. Flow regimes for liquid water transport in a tapered flow channel of proton exchange membrane fuel cells (PEMFCs), J. Power Sources, v. 234, p. $260-271,2013$.

FUDYM, O.; PRADERE, C.; BATSALE, J. C. An analytical two-temperature model for convectiondiffusion in multilayered systems: Application to the thermal characterization of microchannel reactors. Chem. Eng. Science, v. 62, p. 4054 - 4064, 2007.

LI, Y. R.; WANG, S. C.; WU, S. Y.; PENG, L. Asymptotic solution of thermocapillary convection in thin annular two-layer system with upper free surface. Int. J. of Heat and Mass Transfer, v. 52, p. $4769-4777,2009$.

MANCUSI, E.; FONTANA, É; SOUZA, A. A. U.; SOUZA, S. M. A. G. U. Numerical study of twophase flow patterns in the gas channel of PEM fuel cells with tapered flow field design. Int. J. Hydrogen Energy, v. 39, n. 5, 2261 - 2273, 2014.

PELLEW, A.; SOUTHWELL, R. V. On maintained convective motion in a fluid heated from below. Proc. of the Royal Society, v. 176, n. 966, p. 312 - 342, 1940.

PRAKASH, A.; YASUDA, K.; OTSUBO, F.; KUWAHARA, K.; DOI, T. Flow coupling mechanisms in two-layer Rayleigh-Bénard convection. Exp. in Fluids, v. 23, p. 252 - 261, 1997.

RASENAT, S.; BUSSE, F. H.; REHBERG, I. A theoretical and experimental study of double-layer convection. J. of Fluid Mechanics, v. 199, p. 519 - 540, 1989. 